\title{
Anterior gradient 2 as a supervisory marker for tumor vessel normalization induced by anti-angiogenic treatment
}

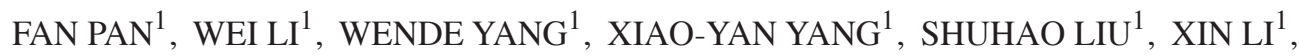 \\ XIAOXU ZHAO ${ }^{1}$, HUI DING ${ }^{1}$, LI QIN ${ }^{2}$ and YUNLONG PAN ${ }^{1}$ \\ ${ }^{1}$ Department of General Surgery, The First Affiliated Hospital of Jinan University; ${ }^{2}$ Department of Histology and Embryology, \\ Medical School of Jinan University, Guangzhou, Guangdong 510632, P.R. China
}

Received February 25, 2017; Accepted May 14, 2018

DOI: $10.3892 / \mathrm{ol} .2018 .8996$

\begin{abstract}
Anti-angiogenic therapy provides transient tumor vascular normalization, which results in a window of opportunity for improvement of radio- or chemotherapy. Biomarkers indicating this window are required for rationalizing anti-angiogenesis. Anterior gradient 2 (AGR2), the majority of which is secreted from tumor cells, is an easily detected plasma protein. In the present study, it was demonstrated that AGR2 could be applied as a biomarker for the supervision of vascular normalization during anti-angiogenic treatment with gold nanoparticles (AuNPs). Nude mice inoculated with SW620 human colorectal cancer cells were treated with AuNPs. Vessel density, pericyte coverage, vessel permeability, tumor hypoxia, tumor growth and AGR2 secretion were detected following treatment with AuNPs at days 0, 4, 6, 9 and 14. Tumor volume and vessel density were reduced, whereas pericyte coverage was increased, and hypoxia and vessel permeability were improved between days 6-9; however, these improvements decreased by day 14 , revealing a time frame for tumor vascular normalization, namely days 4-9, during treatment with AuNPs in mice. AGR2 levels in tumor tissues and plasma were significantly low at day 9, along with vascular normalization; therefore, AGR2 can be used as a potential marker for monitoring tumor vascular normalization during anti-angiogenic treatment.
\end{abstract}

Correspondence to: Professor Yunlong Pan, Department of General Surgery, The First Affiliated Hospital of Jinan University, 612 Huangpu West Avenue, Guangzhou, Guangdong 510632, P.R. China

E-mail: tpanyl@jnu.edu.cn

Abbreviations: AGR2, anterior gradient 2; AuNPs, gold nanoparticles

Key words: anterior gradient 2, gold nanoparticles, anti-angiogenesis, hypoxia, vessel normalization

\section{Introduction}

Anti-angiogenic therapy, initially proposed in Folkman's hypothesis, is applied with the aim of reducing tumor angiogenesis and growth (1). However, excessive anti-angiogenic treatment can increase areas of hypoxia and decrease blood perfusion in tumors, processes partly accountable for the development of radio- or chemotherapy resistance (2). Thus, although the concept of anti-angiogenesis appears to have conflicting results, a new hypothesis proposed that there is a window of opportunity for the transient normalization of abnormal structures and functions of tumor vessels during anti-angiogenic treatment $(3,4)$. These 're-normalized' vessels improved hypoxic conditions within the tumor by increasing blood perfusion and decreasing vascular leakage, rendering chemoand radiotherapy more effective (5-7). Monitoring of vessel normalization is required for the development of improved combination therapies and for strengthening the antitumor effect of anti-angiogenic therapies. So far, data have proposed that by tracking improvements to intratumoral areas of hypoxia during anti-angiogenic therapy, ${ }^{18} \mathrm{~F}$-fluoromisonidazole-PET could identify vascular normalization $(8,9)$. However, high costs and technical requirements continue to limit the wide clinical application of ${ }^{18} \mathrm{~F}$-fluoromisonidazole-PET. Therefore, the aim was to discover reliable and easily detected biomarkers for monitoring the window of tumor vascular normalization during anti-angiogenic therapy in clinical practice.

Anterior gradient 2 (AGR2) is a human ortholog of the Xenopus laevis cement gland protein and a member of the protein disulfide isomerase (PDI) family (10). AGR2 is highly expressed and secreted in malignant cancer types, including breast carcinomas, pancreatic cancer, glioblastoma, prostate cancer and metastatic colorectal cancer (11-18). Additionally, high levels of plasma AGR2 are associated with a poor prognosis in numerous cancer types (19-22). It has been reported that a hypoxic microenvironment induces AGR2 expression in tumor cells, hypoxia inducible factor-1 $\alpha$ (HIF- $1 \alpha$ ) regulated intracellular expression and extracellular secretion of AGR2. Furthermore, overexpressed AGR2 stabilizes HIF-1 $\alpha$ in tumor cells $(18,23)$.

Gold nanoparticles (AuNPs) are a promising anti-angiogenic agent with activity against VEGF and VEGF receptor 2 (VEGFR2). Our previous study showed that, in vitro, AuNPs 
could inhibit VEGF-induced activation of phosphorylated AKT in endothelial cells and disrupt the proliferation or migration of endothelial cells in tumor cell-conditioned medium, which further indicated that AuNPs could lessen the angiogenic tendency of endothelial cells in the tumor microenvironment. Furthermore, it was confirmed that AuNPs are biocompatible and stable, with low cytotoxicity (24-26). Further studies showed that AuNPs would muster in solid tumors, and could penetrate directly into tumor vessels (27-29). Further research ascertained that AuNPs, as a nanoparticle-based drug-delivery system, could deliver other anti-angiogenic agents and reengineer abnormal vessels, resulting in improvements to areas of hypoxia in the tumor and more effective chemotherapy (30).

Because abnormal vasculature cannot supply sufficient oxygen to the tumor, the interstitial tissue remains under hypoxic conditions (5-7). Anti-angiogenic agents reduce hypoxia during the window of tumor vascular normalization; however, extended anti-angiogenic therapy causes excess tumor vessel reduction, which eventually creates more hypoxic areas within the tumor (31). It is hypothesized that AGR2 could detect internal tumor hypoxia, which offered an opportunity to track vascular abnormality and vascular 're-normalization' during anti-angiogenic treatment. In the present study, to elucidate the role of AGR2 as a potential biomarker of vascular normalization during anti-angiogenic treatment, it was analyzed whether AuNPs could normalize tumor vasculature in nude mice that were ectopically transplanted with human metastatic colorectal cancer (SW620) cells, and how AGR2 secretion and expression profiles changed in plasma and tumor microenvironments during treatment.

\section{Materials and methods}

Cell line, mouse xenografts and treatment design. SW620 metastatic colorectal cancer cells were purchased from Shanghai Zzbio Co., Ltd. (Shanghai, China). Cells were maintained at $37^{\circ} \mathrm{C}$ with $5 \% \mathrm{CO}_{2}$ and $95 \%$ humidity, and cultured with Dulbecco's modified Eagle's medium (Gibco; Thermo Fisher Scientific, Inc., Waltham, MA, USA) containing 10\% fetal bovine serum (Gibco; Thermo Fisher Scientific, Inc.) and $1 \%$ penicillin/streptomycin (HyClone; GE Healthcare Life Sciences, Logan, UT, USA). AuNPs were purchased from Shanghai Jie Ning Biotechnology Co., Ltd. (Shanghai, China), stored in light-resistant containers at $4^{\circ} \mathrm{C}$, and the hydrodynamic size distribution of AuNPs was 58.2 $\pm 7.1 \mathrm{~nm}$. Female BALB/c nude mice ( $\mathrm{n}=57,18-22 \mathrm{~g}$ weight, 6-8 weeks old) were purchased from the Guangdong Medical Laboratory Animal Center (Guangdong, China) and maintained under pathogen-free conditions (temperature, $23 \pm 2^{\circ} \mathrm{C}$; light/dark

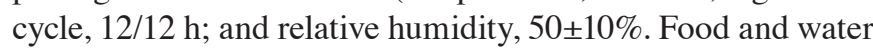
were provided ad libitum. All animal study protocols were approved and conducted in accordance with the guidelines of the Laboratory Animal Ethics Committee of Jinan University (Guangzhou, Guangdong, China). Mouse xenografts were constructed by suspending SW620 metastatic colorectal cancer cells $\left(4 \times 10^{6} / 100 \mu \mathrm{l} / \mathrm{mouse}\right)$ in saline and subcutaneously injecting the solution into the flank. The day when the volume of xenografts reached $175-200 \mathrm{~mm}^{3}$ was designated as day 0 . In the group receiving treatment with AuNPs, the mice were subcutaneously given an injection of $1.3 \mathrm{mg} / \mathrm{kg}$ and the treatment was repeated daily from day 1-6, and then repeated every other day from day 8-14. Mice were weighed and the tumor volume was measured daily. The tumor volumes were measured in two dimensions with calipers and calculated with the formula $\left(\mathrm{L} \mathrm{x} \mathrm{W}^{2}\right) \times 0.5$ ( $\mathrm{L}$ is the length and $\mathrm{W}$ is the width of tumor). Mice were anesthetized with lidocaine (intraperitoneal injection, $10 \mathrm{mg} / \mathrm{kg}$ ). Tumors were harvested and examined on day $0,4,6,9$ and 14 (n=3/time point). Blood samples were collected from the retro-orbital plexus at day $0,2,4,6,9,12$ and 14 (n=6/time point). Control mice were injected with saline.

Plasma AGR2 ELISA. Blood samples were collected in test tubes and immediately centrifuged at 2,000 x g for $15 \mathrm{~min}$ at $4^{\circ} \mathrm{C}$ to obtain plasma samples, following which they were stored at $-40^{\circ} \mathrm{C}$. An ELISA kit for AGR2 (MEXN-H0280; Meixuan Biological Technology Co., Ltd.; Shanghai, China) (URL: http://www.mexnbio.com/mxbio-Products-19629906/) was used to measure the level of serum AGR2, according to the manufacturer's instructions.

Immunofluorescence. The mice were sacrificed and tumors were harvested. The tumor tissues (10-20 $\mu \mathrm{m}$ thick) were fixed in $4 \%$ paraformaldehyde for $24 \mathrm{~h}$ at $4{ }^{\circ} \mathrm{C}$, paraffin-embedded and sectioned. Following on, the tissues were dewaxed in Xylene and rehydrated with graded alcohol. Antigen retrieval was conducted in citric acid buffer (PH 6.0) for $10 \mathrm{~min}$ at $98^{\circ} \mathrm{C}$. Tumor sections were blocked in $2 \%$ normal goat serum (1:200; ProteinTech Group, Inc., Chicago, IL, USA) for $1 \mathrm{~h}$ at room temperature. Then, the sections were incubated overnight at $4^{\circ} \mathrm{C}$ with an anti-cluster of differentiation (CD)-31 antibody (1:500; ab28364; Abcam, Cambridge, UK), an $\alpha$-smooth muscle actin (SMA) antibody (1:100; 14395-1-AP; ProteinTech Group, Inc.), an anti-VEGFR2 antibody (1:100; ab2349; Abcam) and an anti-AGR2 antibody (1:500; EPR20164-278; Abcam). Then those sections were washed and incubated with rhodamine-conjugated goat anti-rabbit IgG-FITC (1:200; sc-2359; Santa Cruz Biotechnology, Inc., Dallas, TX, USA) for $40 \mathrm{~min}$ at room temperature. The nuclei were counterstained using DAPI for $15 \mathrm{~min}$ at room temperature (00-4959-52; Invitrogen; Thermo Fisher Scientific, Inc.), and the tissues were visualized using a fluorescent microscope (x20 to x1,000; Leica DM6000B; Leica Microsystems GmbH, Wetzlar, Germany) at magnification x20. For each section, a total of 8 images were taken of randomly selected fields of view.

Histology and immunohistochemistry. Tumors were dissected and fixed in $10 \%$ formalin solution, embedded in paraffin and sectioned into 3 -mm-thick sections. The remaining tissue sections were cryopreserved in isopentane at $-70^{\circ} \mathrm{C}$. Hematoxylin and eosin (H\&E) staining was performed on 3-mm paraffin-embedded sections by staining nuclei with alum hematoxylin (2 min), washing in running tap water for 5 min, differentiating with $0.3 \%$ acid alcohol for 1 min, rinsing with tap water for min, staining with eosin for 2 min, dehydrating through $95 \%$ alcohol for $10 \mathrm{~min}$ and then 2 incubations with absolute alcohol for $5 \mathrm{~min}$ each. All H\&E procedures were performed at room temperature. A Hypoxyprobe ${ }^{\mathrm{TM}}-1$ 
Plus kit (Hypoxyprobe Inc., Burlington, MA) was used for pimonidazole staining. Mice were intraperitoneally injected with pimonidazole at a dose of $60 \mathrm{mg} / \mathrm{kg}$, and sacrificed after $1 \mathrm{~h}$ to obtain tumor samples. Hypoxyprobe ${ }^{\mathrm{TM}}-1$ adducts were examined with an affinity-purified rabbit IgG polyclonal antibody (1:100; ab208280; Abcam) conjugated with horseradish peroxidase according to the manufacturer's instructions. The images were obtained using a Leica DM6000 B fluorescent microscope (Leica DM6000B) at magnification x20. For each section, a total of 8 images were taken of randomly selected fields of view.

Image analysis. To evaluate vessel density, vessel maturity, vessel integrity, hypoxic areas, necrotic areas and AGR2 expression in tumor tissues, images were obtained of 8 random fields per tumor section at x20 magnification and analyzed with ImageJ software (version 1.48; National Institutes of Health, Bethesda, MD, USA). Vessel density was measured by counting CD31-positive areas per field. The pericyte coverage, one of the most important indicators of vessel maturity was quantified as $\alpha$-SMA staining area/CD31 positive area. The vessel integrity was quantified as the radio of the vessels surrounded by VEGFR2-positive area per field and presented as the VEGFR2 staining area/CD31 positive area. Hypoxic areas were measured by pimonidazole staining areas per field. Necrotic areas were characterized by unclear cell structure and nuclear pyknosis, and calculated as the percentage of necrotic regions compared to the total tumor areas per field by H\&E staining. The necrotic and total areas were quantified by CellProfiler software (version 3.0.0; http://cellprofiler.org/).

Protein extraction and western-blotting assay. RIPA lysis buffer (Beyotime Institute of Biotechnology, Haimen, China) was used to lyse the tumor tissues. BCA protein Assay kits (Takara Biotechnology Co., Ltd., Dalian, China) were used to detect protein concentrations. Proteins (40 $\mu \mathrm{g} / \mathrm{lane})$ were separated via $12 \%$ SDS-PAGE and transferred onto polyvinylidene difluoride membranes. A western-blot assay was conducted for the detection of HIF-1 $\alpha$ and AGR2, using a HIF-1 $\alpha$ antibody $(1: 100 ; 20960-1-A P ;$ ProteinTech Group, Inc.) and an anti-AGR2 antibody (1:500; EPR20164-278; Abcam) as the primary antibody overnight at $4^{\circ} \mathrm{C}$. The internal control protein was used to detect the protein concentrations, along with a pro- $\beta$-actin antibody $(1: 1,000$; ab8226; Abcam). Detection was performed using an enhanced chemiluminescent kit (20148; GE Healthcare Life Sciences). An enhanced chemiluminescence detection system (ChemiDoc XRS; Bio-Rad Laboratories, Inc., Hercules, CA, USA) and Quantity One software (version 4.62; Bio-Rad Laboratories, Inc.) were used to visualize and quantify the resultant bands.

Statistical analysis. All of the data were displayed as the mean \pm standard error. A Student's t-test was the principal statistical test for two-group comparisons; one-way analysis of variance with a Bonferroni test for pairwise comparison was used for multiple group comparisons. All statistical analyses were performed using GraphPad Prism 5.0 (GraphPad Software, Inc., La Jolla, CA, USA). A value of $\mathrm{P}<0.05$ was considered to indicate a statistically significant difference.

\section{Results}

AuNPs delays tumor growth. To examine whether treatment with AuNPs could result in vessel normalization in a SW620 xenograft tumor model, mice were inoculated with cancer cells and injected subcutaneously with $1.3 \mathrm{mg} / \mathrm{kg}$ AuNPs when the tumor volume reached $175-200 \mathrm{~mm}^{3}$. The treatments were repeated daily from day 1 to day 6 , and then repeated every other day from days 8-14. Plasma collection was conducted at days $0,2,4,6,9,12$ and 14, and tumor harvest was conducted at days $0,4,6,9$ and 14 (Fig. 1A). There was no significant different in the body weights of mice treated with AuNPs, compared with those of mice treated with saline (Fig. 1B); whereas, tumors in AuNPs-treated mice exhibited a considerably slower growth pattern as compared with those in saline-treated mice, indicating that AuNPs have an antitumor effect on the SW620 xenograft tumor models $(\mathrm{P}=0.02$; Fig. 1B).

AuNPs decreases vessel density and reconstructs vessel morphology. To demonstrate the tumor vessel density after treatment with AuNPs, tumor tissues were stained with the endothelial cell marker CD31. A significant decrease was exhibited in AuNPs-treated tumor vessel density at day 6 $(\mathrm{P}=0.031)$ and $9(\mathrm{P}=0.042)$ (Fig. 2A). It was revealed that vessel density decreased with AuNP treatment by day $9(\mathrm{P}=0.019$; Fig. 2B); however, AuNPs-treated tumor vessels reversely increased at day 14 and had no statistic significant difference with day 0 (Fig. 2B). Furthermore, CD31 staining also revealed that vessel in saline-treated tumor was collapsed on the peripheral region, but dilated and discontinued in the central region, compared with vessel in AuNPs-treated tumor at day 9, which was well-organized and -structured (Fig. 2C). On day 9 after AuNPs treatment, vessel density in AuNPs-treated tumor was less abundant than that in saline-treated tumor ( $\mathrm{P}=0.042$; Fig. 2C). These results suggested that AuNPs normalized vascular growth, as demonstrated by a reduction in the number of abnormal tumor vessels and vessel density.

AuNPs increases pericyte adhesion and maintains vessel maturity. To evaluate whether AuNPs could normalize tumor vessel morphology, vessel maturity in AuNPs-treated tumors was studied. Tumor tissues were double-stained with CD31 and the pericyte marker $\alpha$-SMA. There were $\alpha$-SMA-positive cells observed at the CD31-positive area in SW620 xenograft tumors prior to the AuNPs treatment (day 0). After treatment with AuNPs, the $\alpha$-SMA-positive cells appeared to be constantly distributed and had closer contact with CD31-positive areas in the tumor at days 6 and 9; however, at day 14, as the CD31-positive area decreased, the number of $\alpha$-SMA-positive cells also decreased (Fig. 3A). Mature vessels were defined as those with $\alpha$-SMA-positive cell adhesion, and the vessel maturity ratio was calculated as the $\alpha$-SMA-positive area to the CD31-positive area, at the same scale (32). The quantity of $\alpha$-SMA-positive cells increased at days $6(\mathrm{P}=0.39)$ and $9(\mathrm{P}=0.027)$, but decreased again at day $14(\mathrm{P}=0.037)$ during the treatment with AuNPs. Furthermore, the vessel maturity ratio was significantly increased, due to the decrease of CD31 staining and increase of $\alpha$-SMA staining at day $9(\mathrm{P}=0.019)$, although it was not statistically significant 


\section{A}
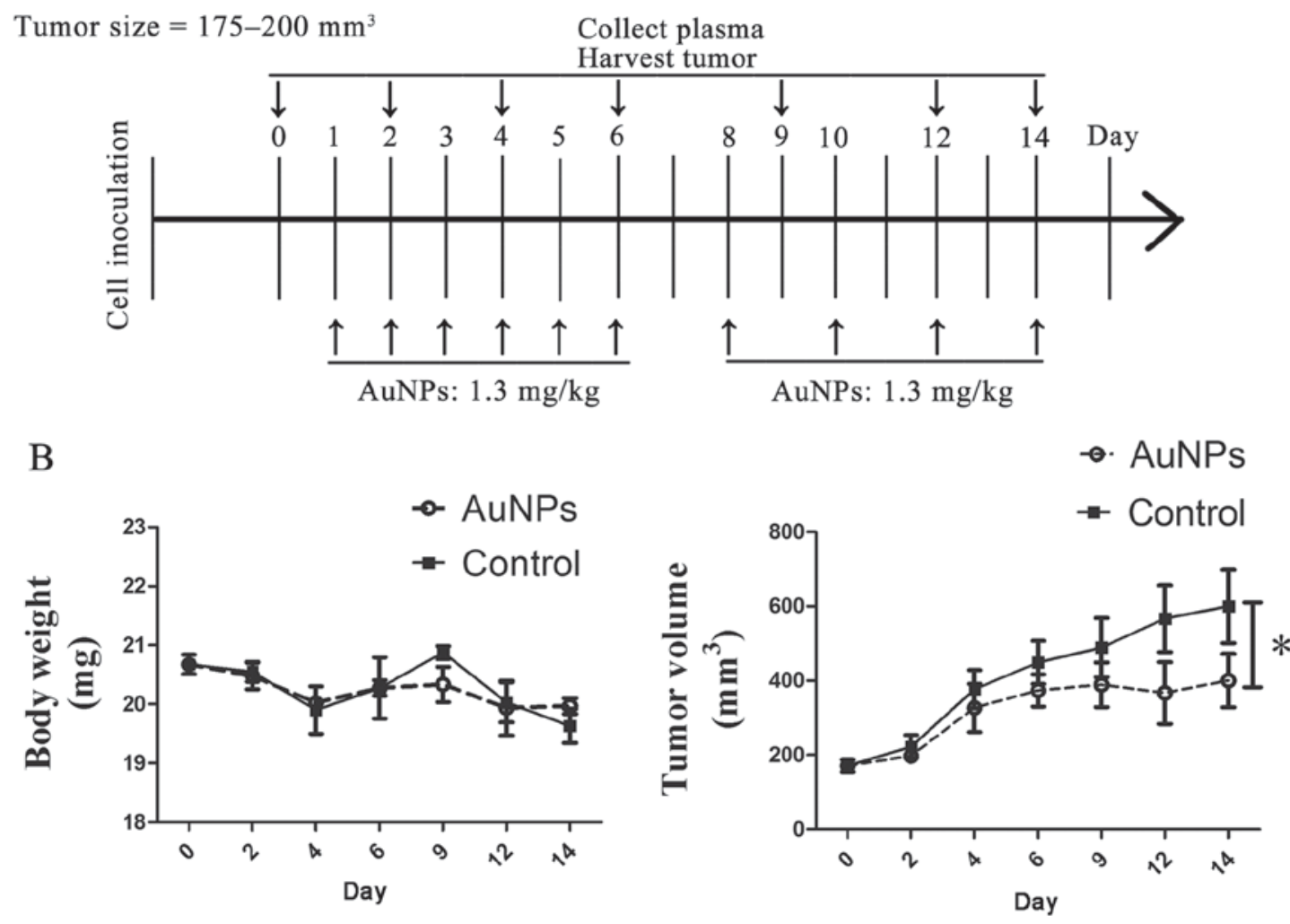

Figure 1. AuNPs delayed tumor growth. (A) Schematic of the study design. SW620-bearing mice were treated daily from day 1-6, and then every other day from day 8-14, with AuNPs $\left(1.3 \mathrm{mg} / \mathrm{kg}\right.$ ) when the tumor size reached $175-200 \mathrm{~mm}^{3}$ (designated as day 0 and control mice). Control mice were injected with saline. Tumors were harvested and examined at days $0,4,6,9$ and 14 ( $\mathrm{n}=3$ per time point). Blood samples were collected from the retro-orbital plexus at days 0 , 2, 4, 6, 9, 12 and 14 (n=6 per time point). (B) Quantitative evaluation of body weight and tumor growth after treatment with AuNPs and saline. AuNPs treatment allowed retention of comparable body weights $(\mathrm{P}>0.05)$ but delayed tumor growth $(* \mathrm{P}<0.05)$. AuNPs, gold nanoparticles.

at day 14 (Fig. 3B and C). These results indicated that AuNPs stimulated the expression of $\alpha$-SMA-positive cells that accumulated near endothelial cells and resulted in the maturity of tumor vessels.

AuNPs reduces VEGFR2 expression and alleviates vessel hyperpermeability. To further explore the morphological changes of tumor blood vessels during AuNP treatment, vessel hyperpermeability was also studied. It has been reported that VEGFR2 expression levels in endothelial cells are associated with vessel hyperpermeability, and that high expression of VEGFR2 is responsible for tumor vessel leakage (33). It is considered that vessel hyperpermeability is represented by the ratio of VEGFR2-positive areas to CD31-positive areas, at the same scale. The expression level of VEGFR2 was diminished after treatment with AuNPs at day $9(\mathrm{P}=0.042)$, but increased again at day $14(\mathrm{P}=0.026)$, remaining at a high level. Additionally, the ratio of the VEGFR2-positive area to the CD31-positive area was lower at day $9(\mathrm{P}=0.046)$ and became significantly higher again at day $14(\mathrm{P}=0.019$; Fig. 4A and B). These results indicated that AuNPs inhibit VEGFR2 expression in tumor endothelial cells and alleviate vessel hyperpermeability.

AuNPs improves areas of tumor hypoxia and preserves tissue viability. To verify functional changes to tumor vessels that followed morphological changes induced by AuNP treatment, tumor oxygenation as an assessment for vessel function was utilized. Pimonidazole staining was conducted to evaluate tumor hypoxia, which demonstrated that AuNPs reduced tumor hypoxia by days 4 and 9 , particularly by day 9 ; however, the tumor hypoxia increased again after treatment for 14 days $(\mathrm{P}=0.31)$. Furthermore, $\mathrm{H} \& \mathrm{E}$ staining demonstrated that, at days 0, 4, 6 and 9, there was no significant difference in viability among the AuNP-treated tumor tissues; however, compared with day 0 , there was an increase in the size of necrotic areas by day $14(\mathrm{P}=0.028$; Figs. $5 \mathrm{~A}$ and $\mathrm{B})$. Hypoxia is mediated by HIF-1 $\alpha$ (34); thus to further identify improvements to hypoxia, expression of HIF-in AuNP-treated tumor tissue was assessed. The data demonstrated that expression of HIF-1 $\alpha$ decreased by days 6 and 9, but increased by day 14 following AuNPs treatment ( $\mathrm{P}=0.043$; Fig. 5C). Reduced tumor hypoxia indicated that AuNPs can normalize tumor vessels and improve vessel function.

AuNPs abates AGR2 expression during the window of vessel normalization. AGR2 was reported to increase in tumor cells under hypoxia, and be regulated by HIF-1 $\alpha(18,23)$. In the present study, tumor hypoxia observably improved. To investigate whether AGR2 could be used as biomarker to detect AuNP-treated tumor hypoxia improvement during vessel normalization, the AGR2 expression changes in tumor 
A

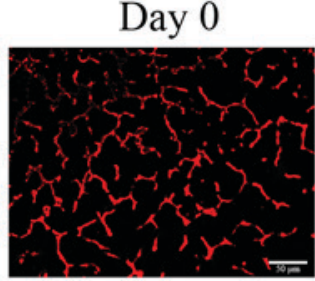

B

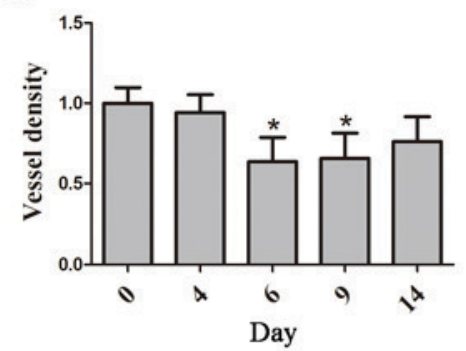

Day 4

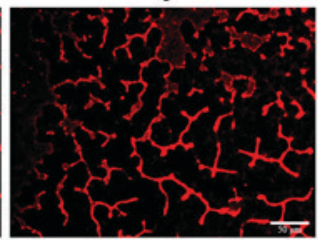

C
Day 6

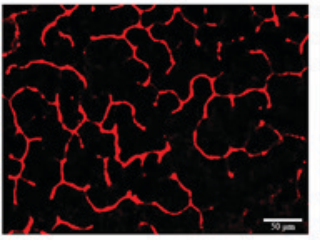

Day 0

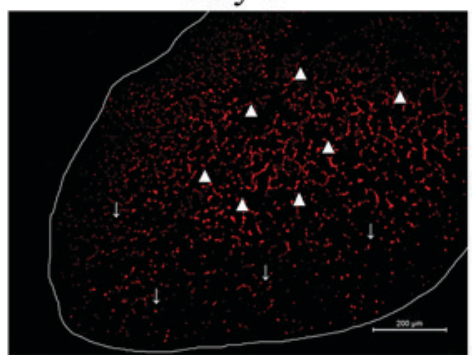

Day 9

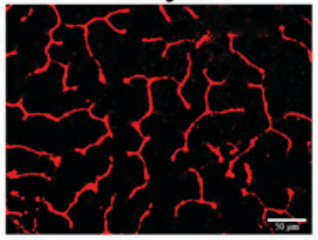

Day 9
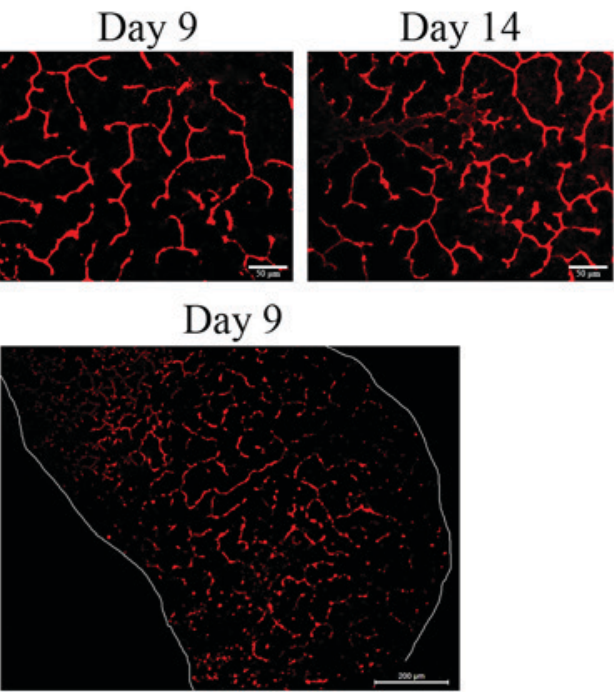

Figure 2. Gold nanoparticles (AuNPs) decreased vessel density and reconstructed vessel morphology. (A) CD31 staining of SW620 tumor from control (day 0) and AuNP-treated mice (days 4, 6, 9 and 14). Tumor tissues were stained with an anti-CD31 antibody (red). Scale bars: $50 \mu \mathrm{m}$. (B) Quantification of vessel density. Eight random fields were analyzed. Vessel density were measured and presented as CD31-positive areas per field. Relative CD31 levels in tumor at days 6 and 9 vs. controls ( $\left.{ }^{*} \mathrm{P}<0.05\right)$. (C) Representative images of SW620 tumor vessels (days 0 and 9). Tumor vessel in saline-treated tumor (day 0) was collapsed (arrows) on the peripheral region but dilated and discontinued (triangles) in the central region. Vessels in AuNPs-treated tumors (day 9) were well organized and structured. Scale bars, $200 \mu \mathrm{m}$. AuNPs, gold nanoparticles; CD, cluster of differentiation.

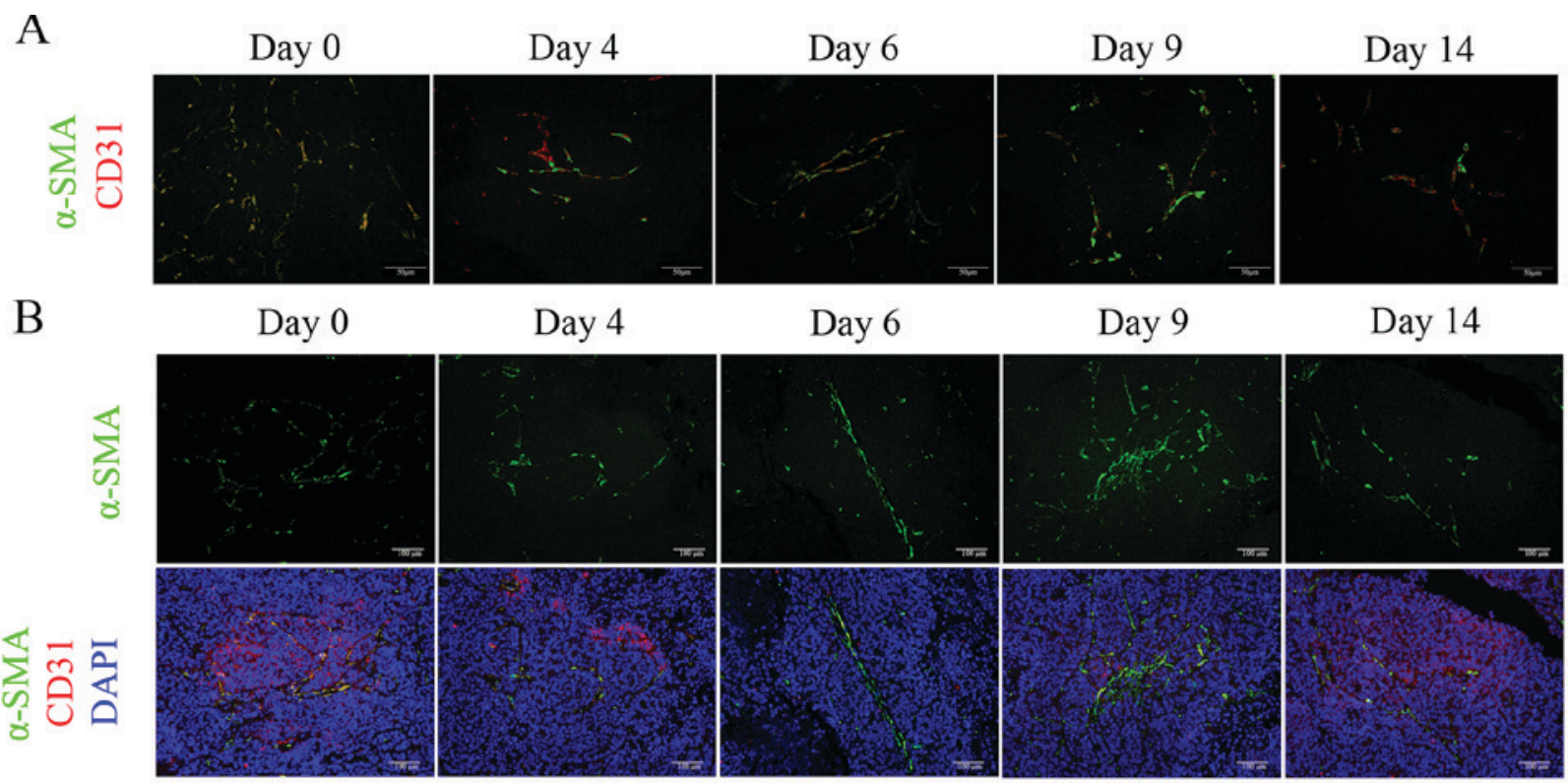

C
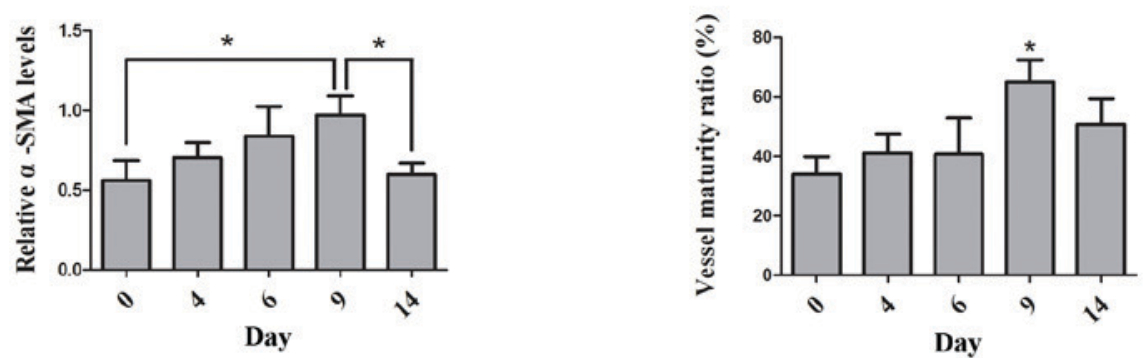

Figure 3. AuNPs increased pericyte adhesion and maintained vessel maturity. (A) Tumor tissues from control mice (day 0) and AuNPs-treated mice (days 4 , 6, 9 and 14) were stained with an anti-cluster of differentiation (CD) 31 antibody (red) and an anti- $\alpha$-smooth muscle actin (SMA) antibody (green). Scale bars: $50 \mu \mathrm{m}$. (B) Upper panel, representative images of $\alpha$-SMA (green) in tumors from control mice and AuNPs-treated mice. Lower panel, merge images of $\alpha$-SMA (green), CD31 (red) and DAPI (blue). Scale bars, $100 \mu \mathrm{m}$. (C) The $\alpha$-SMA positive area was measured and presented as the relative value. The relative $\alpha$-SMA levels in tumor with AuNPs treatment (day 9) vs. controls ( $\mathrm{P}<0.05$ ). The relative $\alpha$-SMA levels at day 9 vs. those at day 14 ("P<0.05). Vessel maturity ratio is presented as the ratio of the $\alpha$-SMA positive area to the vessel density. The relative vessel maturity ratio in day 9 vs. controls ( $\mathrm{P}<0.05$ ). AuNPs, gold nanoparticles; SMA, smooth muscle actin; CD, cluster of differentiation. 
A
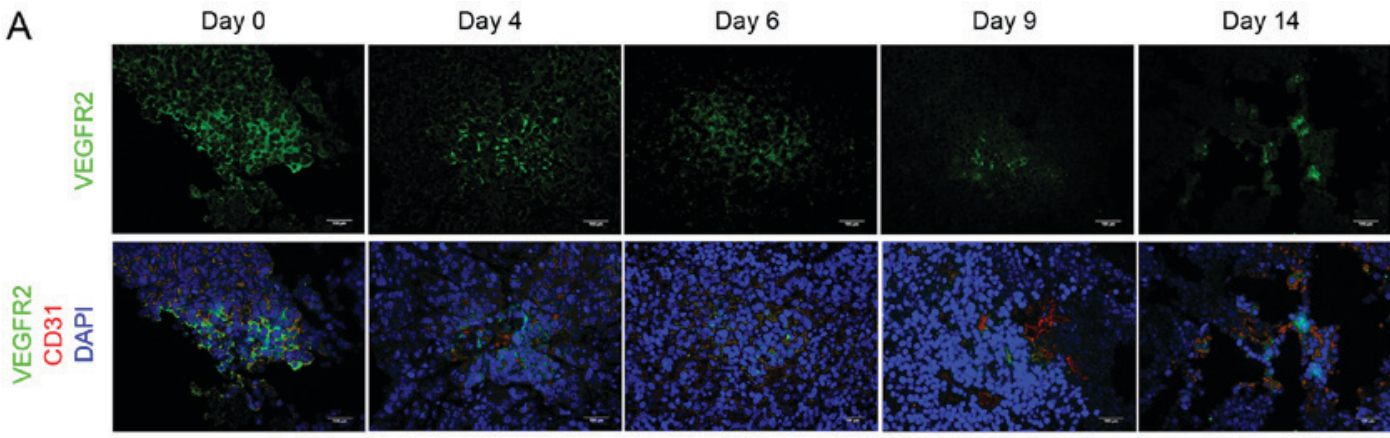

B
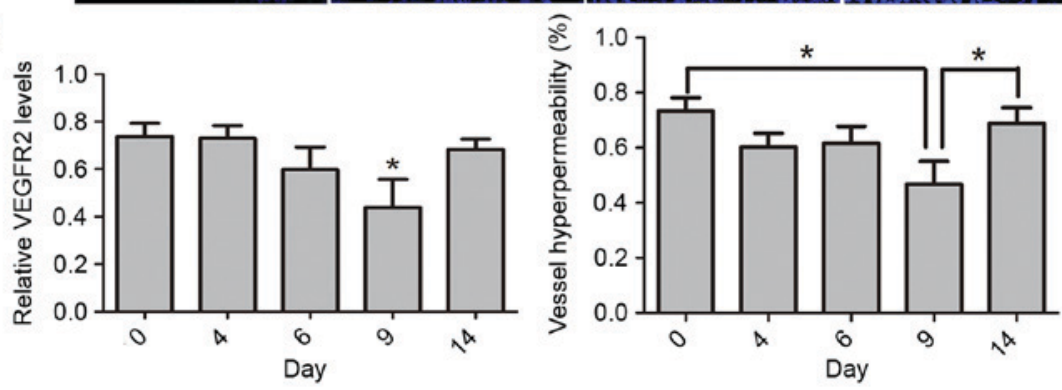

Figure 4. AuNPs reduced VEGFR2 expression and alleviated vessel hyperpermeability. Tumor tissues from control mice (day 0) and AuNPs-treated mice (days 4, 6,9 and 14) were stained with anti-cluster of differentiation (CD) 31 antibody (red) and anti-VEGFR2 antibody (green). (A) Upper panel, representative images of VEGFR2 (green) in tumors from control mice and AuNPs-treated mice. Lower panel, merge images of VEGFR2 (green), CD31 (red) and DAPI (blue). Scale bars, $100 \mu \mathrm{m}$. (B) VEGFR2 positive area was measured and presented as the relative value. Relative VEGFR2 levels in AuNP-treated tumors (day 9) vs. controls ( $\mathrm{P}<0.05)$. Vessel hyperpermeability is presented as the ratio of the VEGFR2 positive area to vessel density. Vessel hyperpermeability at day 9 vs. controls ( $\mathrm{P}<0.05)$. AuNPs, gold nanoparticles; CD, cluster of differentiation; VEGFR2, vascular endothelial growth factor receptor 2.
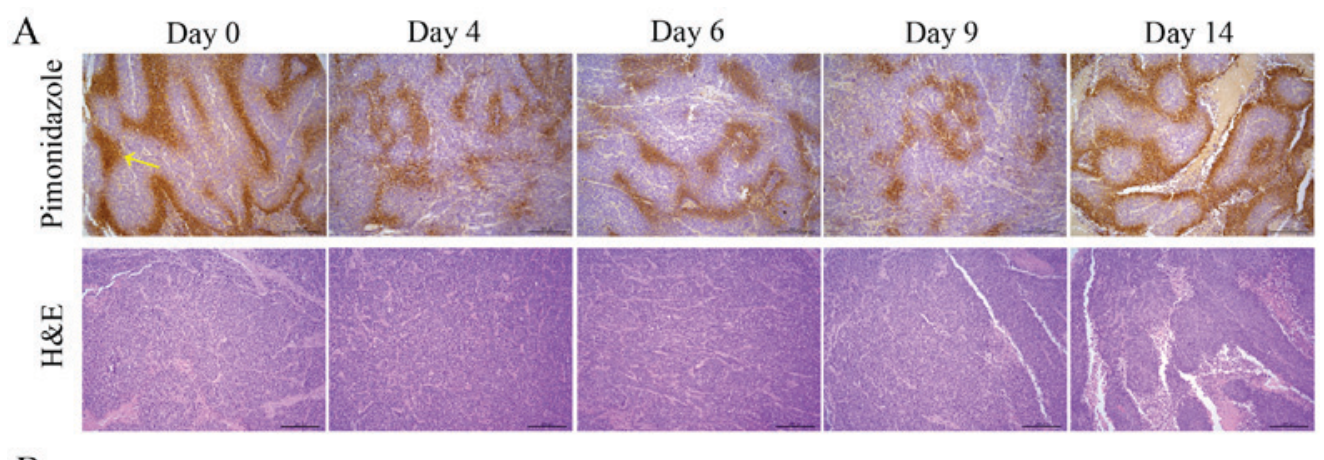

B
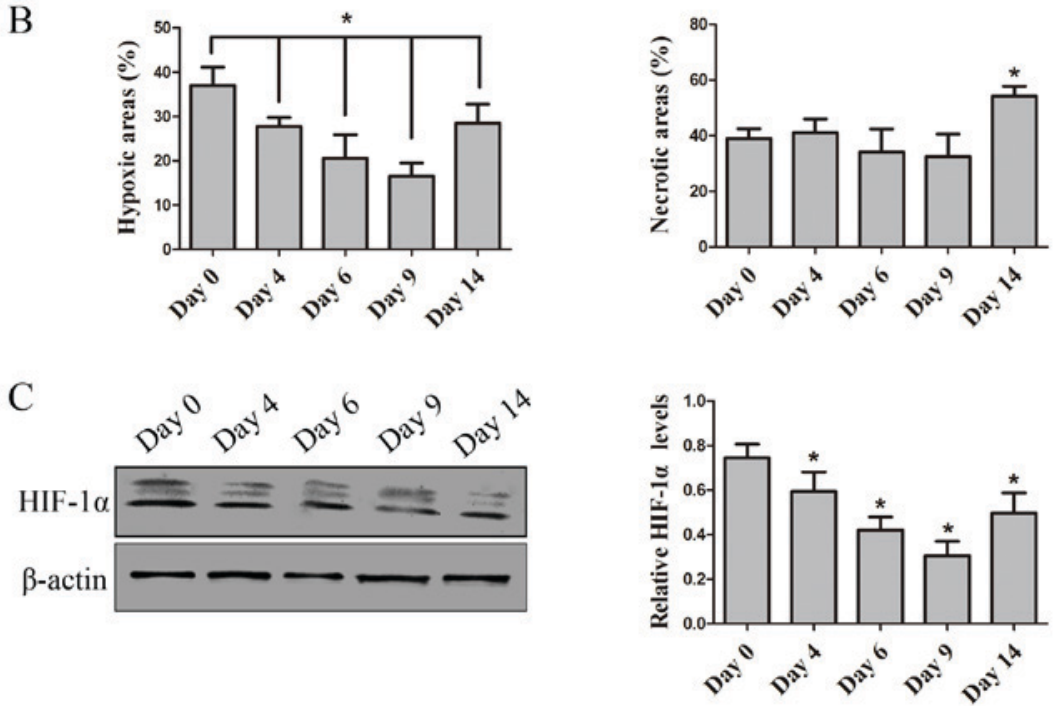

Figure 5. AuNPs improved tumor hypoxia and preserved tissue viability. (A) Upper panel, pimonidazole staining of tumor tissues from control mice (day 0) and AuNPs-treated mice (days 4, 6,9 and 14). The arrow highlights part of the pimonidazole staining positive area. Lower panel, H\&E staining of tumor tissues. Scale bars, $200 \mu \mathrm{m}$. (B) Hypoxic areas were measured and presented as the relative values. Hypoxic areas at different time points during the treatment (AuNPs-treated tumors vs. controls; ${ }^{*} \mathrm{P}<0.05$ ). The necrotic areas in AuNPs-treated tumors vs. controls ( $\left.\mathrm{P}<0.05\right)$. (C) Western blot analysis for HIF-1 $\alpha$ expression in saline- and AuNPs-treated tumor tissues. $\beta$-actin was used as the internal control. HIF-1 $\alpha$ expression of tumor tissues from AuNPs-treated mice vs. control mice ("P<0.05). H\&E, hematoxylin and eosin; AuNPs, gold nanoparticles; HIF-1 $\alpha$, hypoxia-inducible factor-1 $\alpha$. 
A
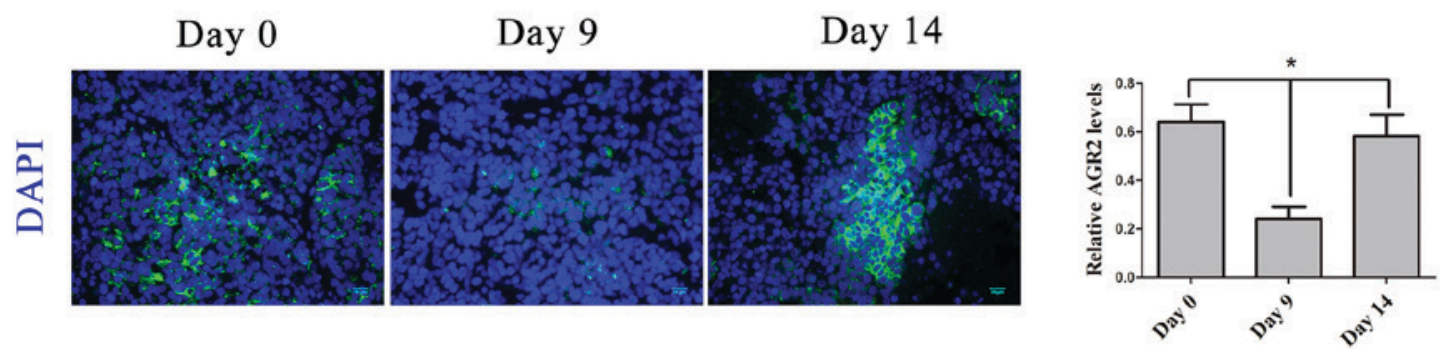

B

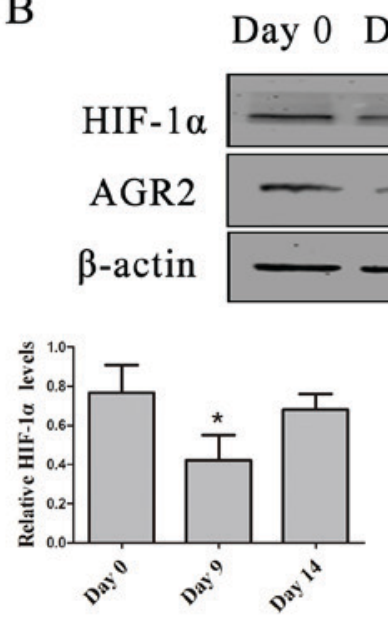

Day 9 Day 14
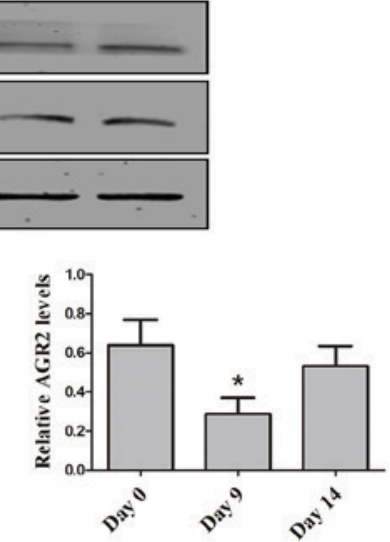

C

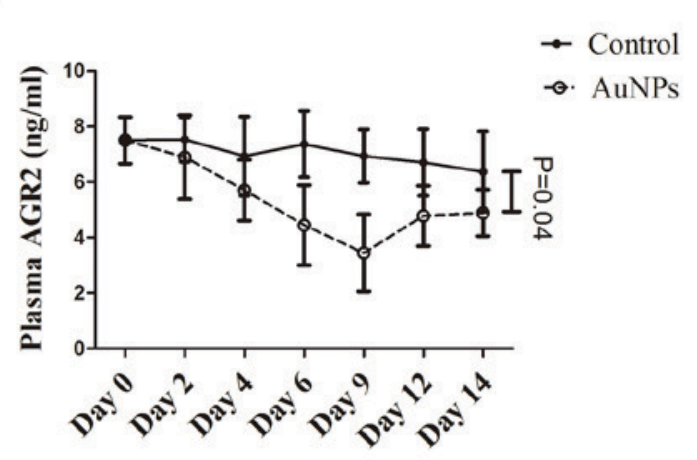

Figure 6. AuNPs abated AGR2 expression during the window of vessel normalization. Tumor tissues from control mice (day 0) and AuNPs-treated mice (days 9 and 14) were stained with an anti-AGR2 antibody (green). (A) Merge images of AGR2 (green) and DAPI (blue). AGR2 expression in tumor from AuNPs-treated mice vs. control mice ( $\mathrm{P}<0.05)$. (B) Western blot analyses for HIF-1 $\alpha$ and AGR2 expression in saline- and AuNPs-treated tumor tissues. $\beta$-actin was used as the internal control. HIF-1 $\alpha$ and AGR2 expression in tumor tissues from AuNPs-treated mice (days 9 and 14) vs. control mice (day 0) $\left({ }^{*} \mathrm{P}<0.05\right)$. (C) Plasma AGR2 in AuNPs-treated mice (day 9) vs. control mice $\left({ }^{*} \mathrm{P}<0.05\right)$. AuNPs, gold nanoparticles; HIF-1 $\alpha$, hypoxia-inducible factor-1 $\alpha$; AGR2, anterior gradient 2.

tissue and plasma following AuNP-treatment was assessed. AGR2 protein expression in tumor tissue decreased by day 9 , but increased again by day $14(\mathrm{P}=0.027$; Fig. 6A). There was significant decrease in AGR2 protein expression by day 9, similar to changes in HIF-1 $\alpha$ protein levels in tumor tissues. AGR2 protein expression increased again by day 14, after AuNP-treatment ( $\mathrm{P}=0.046$; Fig. 6B). Furthermore, plasma AGR2 levels were significantly decreased by day 9 , though increased again by day $14(\mathrm{P}=0.044$; Fig. 6C). These data demonstrate that AGR2, regulated by HIF-1 $\alpha$, could be a supervisory biomarker to detect vessel normalization induced by AuNPs.

\section{Discussion}

Anti-angiogenic agents induce a transient window of vascular normalization where both chemotherapy and radiotherapy achieve a better curative effect $(3,4)$. Direct morphological changes were observed using histological methods, vessel function detection by PET-CT and dynamic MRI, particularly applied to the monitoring of tumor vessel normalization $(6,8)$; however, these methods were neither invasive or expensive, which restricted the combination of anti-angiogenic therapy and chemotherapy in clinic. A reliable and easily detected biomarker was required during anti-angiogenic therapy to identify a suitable time for antitumor treatment.
In the present study, the practicability of AGR2 as a marker to detect tumor vascular normalization after treatment with AuNPs was analyzed. Previous studies indicated that AuNPs interrupted angiogenesis via various pathways, including VEGF and VEGFR2 in vitro $(3,4)$. AuNPs were also reported to be biocompatible and stable, with low cytotoxicity (24-26). In the present study, mice were treated with $1.3 \mathrm{mg} / \mathrm{kg}$ AuNPs daily from day 0-6, which was then repeated every other day from day 8-14. It was revealed that an SW620-inoculated xenograft model was adequate for monitoring the vascular normalization induced by AuNPs. It was demonstrated that AGR2 could be used as a potential marker for observing vessel normalization during AuNP-mediated anti-angiogenesis.

To investigate whether AuNPs have the ability to modify vessel structure, the vessel density, pericyte coverage and vessel hyperpermeability after AuNPs treatment were studied. The results demonstrated that tumor vessel density was significantly reduced at day 6 and 9 after AuNPs treatment. Pericyte coverage increased by day 9 in AuNPs-treated tumors. The vessel maturity ratio significantly increased by day 9 , probably due to reduction of abnormal tumor vessels and increases in pericyte coverage after AuNPs treatment. It was also demonstrated that AuNPs significantly inhibited VEGFR2 expression at day 9, resulting in lower vessel hyperpermeability in the tumor tissue. To investigate the functional improvement that followed structure changes in the tumor 
vessels after AuNPs treatment, tumor hypoxia changes were analyzed. Tumor hypoxia was reduced at days 4, 6 and 9 after AuNPs treatment, with improvement was observed at day 9. In addition, there was no significant difference in the necrosis rate of the tumor tissue at days $0,4,6$ and 9, but there was a slight increase observed at day 14 . The data corroborated that tumor vessel normalization could be induced by anti-angiogenic treatment $(3,4)$.

Using histology and functional methodology, it was demonstrated that anti-angiogenic treatment with AuNPs provided vascular normalization in the tumor at day 9. Based on these results, the expression levels of AGR2 in tumor tissue were investigated. It appeared that AGR2 had lower expression, along with a decreased hypoxic area in the tumor tissue after treatment with AuNPs for 9 days, and, at day 14, an increase in AGR2 expression and hypoxia was observed in the tumor tissue. These results are concordant with those of previous studies, which identified that AGR2 was overexpressed in hypoxic areas and was regulated by $\mathrm{HIF}-1 \alpha(3,4)$. Furthermore, plasma AGR2 levels in mice treated with AuNPs were monitored. AGR2 levels decreased following treatment with AuNPs and reached the lowest point at day 9; however, plasma AGR2 levels increased again by day 14, parallel to the development of tumor hypoxia. The data illustrate that AuNPs-mediated AGR2 expression could monitor the improvement of hypoxia in anti-angiogenic therapy and plasma AGR2 levels could be a supervisory marker for the window of vascular normalization.

Overall, the data illustrate that treatment with AuNPs provides a transient time window of vascular normalization, which improved pericyte coverage, vessel hyperpermeability and tumor hypoxia. Furthermore, AuNPs may suppress AGR2 expression in the window of vascular normalization via improving the hypoxic tumor microenvironment. Excessive treatment with AuNPs indirectly, through hypoxia regulation, stimulated AGR2 to be expressed once again in the tumor; therefore, AGR2 may be a potential monitor of vascular normalization during AuNPs treatment. A study could be conducted to develop a more practical and rational application for AuNPs treatment combined with chemotherapeutic agents; however, whether AuNPs are directly involved in regulating AGR2 expression remains unknown, so further study is required to analyze the reliability of AGR2 expression levels in monitoring vessel normalization induced by other anti-angiogenic agents, including bevacizumab, dovitinib and endostatin. Furthermore, plasma AGR2 levels are known to be a blood-based biomarker for metastasis and recurrence in numerous malignant tumors, including ovarian and pancreatic cancer, and lung adenocarcinoma $(35,36)$. Peripheral blood AGR2 levels in patients with breast cancer, papillary thyroid carcinoma or metastatic colorectal cancer are associated with tumor progression and prognosis (12,37-40). Therefore, the clinical applications of AGR2 expression for identifying vessel normalization in tumors during anti-angiogenic treatment are viable for future work.

\section{Acknowledgements}

Not applicable.

\section{Funding}

This work was supported by the National Natural Science Foundation of China (grant no., 81472849), the Guangdong Natural Science Research (grant no., 2014A030313383) and the Guangdong High-level University Construction Fund for Jinan University (grant no., 88016013034).

\section{Availability of data and materials}

All data generated or analyzed during this study are included in this published article.

\section{Authors' contributions}

FP, WL and WY conducted the study and performed the statistical analysis. FP and YP performed the study design and drafted the manuscript. All the authors participated in the discussion, and read and approved the final manuscript.

\section{Ethics approval and consent to participate}

All procedures involving animals were approved by the Ethics Committee of Jinan University. All animal studies also comply with the ARRIVE guidelines and the AVMA euthanasia guidelines 2013 .

\section{Patient consent for publication}

Not applicable.

\section{Patient consent for publication}

The authors declare that they have no competing interests.

\section{References}

1. Folkman J: Tumor angiogenesis: Therapeutic implications. N Engl J Med 285: 1182-1186, 1971.

2. Jain RK: Anti-angiogenesis strategies revisited: From starving tumors to alleviating hypoxia. Cancer Cell 26: 605-622, 2014.

3. Jain RK: Normalization of tumor vasculature: An emerging concept in antiangiogenic therapy. Science 307: 58-62, 2005.

4. Jain RK: Normalizing tumor vasculature with anti-angiogenic therapy: A new paradigm for combination therapy. Nat Med 7: 987-989, 2001.

5. Cobleigh MA, Langmuir VK, Sledge GW, Miller KD, Haney L, Novotny WF, Reimann JD and Vassel A: A phase I/II dose-escalation trial of bevacizumab in previously treated metastatic breast cancer. Semin Oncol 30 (5 Suppl 16): S117-S124, 2003.

6. Goel S, Duda DG, Xu L, Munn LL, Boucher Y, Fukumura D and Jain RK: Normalization of the vasculature for treatment of cancer and other diseases. Physiol Rev 91: 1071-1121, 2011.

7. Carmeliet $P$ and Jain RK: Molecular mechanisms and clinical applications of angiogenesis. Nature 473: 298-307, 2011.

8. Hernández-AgudoE,MondejarT,Soto-MontenegroML,MegíasD, Mouron S, Sanchez J, Hidalgo M, Lopez-Casas PP, Mulero F, Desco M et al: Monitoring vascular normalization induced by antiangiogenic treatment with $18 \mathrm{~F}$-fluoromisonidazole-PET. Mol Oncol 10: 704-718, 2016.

9. Bao X, Wang MW, Luo JM, Wang SY, Zhang YP and Zhang YJ: Optimization of early response monitoring and prediction of cancer anti-angiogenesis therapy via noninvasive PET molecular imaging strategies of multifactorial bioparameters. Theranostics 6: 2084-2098, 2016. 
10. Park SW, Zhen GH, Verhaeghe C, Nakagami Y, Nguyenvu LT, Barczak AJ, Killeen N and Erle DJ: The protein disulfide isomerase AGR2 is essential for production of intestinal mucus. Proc Natl Acad Sci USA 106: 6950-6955, 2009.

11. Liu D, Rudland PS, Sibson DR, Platt-Higgins A and Barraclough R: Human homologue of cement gland protein, a novel metastasis inducer associated with breast carcinomas. Cancer Res 65: 3796-3805, 2005.

12. Fritzsche FR, Dah E, Pahl S, Burkhardt M, Luo J, Mayordomo E, Gansukh T, Dankof A, Knuechel R, Denkert C, et al: Prognostic relevance of AGR2 expression in breast cancer. Clin Cancer Res 12: 1728-1734, 2006 .

13. Innes HE, Liu D, Barraclough R, Davies MP, O'Neill PA, Platt-Higgins A, de Silva Rudland S, Sibson DR and Rudland PS Significance of the metastasis-inducing protein AGR2 for outcome in hormonally treated breast cancer patients. Br J Cancer 94: 1057-1065, 2006.

14. Wang Z, Hao Y and Lowe AW: The adenocarcinoma-associated antigen, AGR2, promotes tumor growth, cell migration, and cellular transformation. Cancer Res 68: 492-497, 2008.

15. Ramachandran V, Arumugam T, Wang HM and Logsdon CD: Anterior gradient 2 is expressed and secreted during the development of pancreatic cancer and promotes cancer cell survival. Cancer Res 68: 7811-7818, 2008.

16. Zhang YX, Ali TZ, Zhou H, D'Souza DR, Lu Y, Jaffe J, Liu Z, Passaniti A and Hamburger AW: ErbB3 binding protein 1 represses metastasis-promoting gene anterior gradient protein 2 in prostate cancer. Cancer Res 70: 240-248, 2010.

17. Gao H, Xu XC, Chen B, Wang F, Zhang W, Geng H and Wang Y: Anterior gradient 2: A new target to treat colorectal cancer. Med Hypotheses 80: 706-708, 2013.

18. Hong XY, Wang $\mathrm{J}$ and $\mathrm{Li} \mathrm{Z}$ : AGR2 expression is regulated by HIF-1 and contributes to growth and angiogenesis of glioblastoma. Cell Biochem Biophys 67: 1487-1495, 2013.

19. Chevet E, Fessart D, Delom F, Mulot A, Vojtesek B, Hrstka R, Murray E, Gray T and Hupp T: Emerging roles for the pro-oncogenic anterior gradient-2 in cancer development. Oncogene 32: 2499-2509, 2013.

20. Brychtova V, Vojtesek B and Hrstka R: Anterior gradient 2: A novel player in tumor cell biology. Cancer Lett 304: 1-7, 2011

21. Valladares-Ayerbes M, Blanco-Calvo M, Reboredo M, Lorenzo-Patiño MJ, Iglesias-Díaz P, Haz M, Díaz-Prado S, Medina V, Santamarina I, Pértega S, et al: Evaluation of the adenocarcinoma-associated gene $A G R 2$ and the intestinal stem cell marker $L G R 5$ as biomarkers in colorectal cancer. Int $\mathrm{J}$ Mol Sci 13: 4367-4387, 2012.

22. Xue H, Lü B, Zhang J, Wu M, Huang Q, Wu Q, Sheng H, Wu D, $\mathrm{Hu} \mathrm{J}$ and Lai M: Identification of serum biomarkers for colorectal cancer metastasis using a differential secretome approach. J Proteome Res 9: 545-555, 2010.

23. Li Z, Zhu Q, Hu L, Chen H, Wu Z and Li D: Anterior gradient 2 is a binding stabilizer of hypoxia inducible factor- $1 \alpha$ that enhances $\mathrm{CoCl}_{2}$-induced doxorubicin resistance in breast cancer cells. Cancer Sci 106: 1041-1049, 2015.

24. Pan Y, Wu Q, Liu R, Shao M, Pi J, Zhao X and Qin L: Inhibition effects of gold nanoparticles on proliferation and migration in hepatic carcinoma-conditioned HUVECs. Bioorg Med Chem Lett 24: 679-684, 2014

25. Pan Y, Wu Q, Qin L, Cai J and Du B: Gold nanoparticles inhibit VEGF165-induced migration and tube formation of endothelial cells via the Akt pathway. Biomed Res Int 2014: 418624, 2014.

26. Pan Y, Ding H, Qin L, Zhao X, Cai J and Du B: Gold nanoparticles induce nanostructural reorganization of VEGFR2 to repress angiogenesis. J Biomed Nanotechnol 9: 1746-1756, 2013.
27. Ernsting MJ, Murakami M, Roy A and Li SD: Factors controlling the pharmacokinetics, biodistribution and intratumoral penetration of nanoparticles. J Control Release 172: 782-794, 2013.

28. Li Y, Lian Y, Zhang LT, Aldousari SM, Hedia HS, Asiri SA and Liu WK: Cell and nanoparticle transport in tumour microvasculature: The role of size, shape and surface functionality of nanoparticles. Interface Focus 6: 20150086, 2016.

29. Baban DF and Seymour LW: Control of tumour vascular permeability. Adv Drug Deliv Rev 34: 109-119, 1998.

30. Li W, Zhao X, Du B, Li X, Liu S, Yang XY, Ding H, Yang W, Pan F, Wu X, et al: gold nanoparticle-mediated targeted delivery of recombinant human endostatin normalizes tumour vasculature and improves cancer therapy. Sci Rep 6: 30619, 2016.

31. Franco M, Man S, Chen L, Emmenegger U, Shaked Y, Cheung AM, Brown AS, Hicklin DJ, Foster FS and Kerbel RS: Targeted anti-vascular endothelial growth factor receptor-2 therapy leads to short-term and long-term impairment of vascular function and increase in tumor hypoxia. Cancer Res 66: 3639-3648, 2006

32. Yonenaga Y, Mori A, Onodera H, Yasuda S, Oe H, Fujimoto A, Tachibana $\mathrm{T}$ and Imamura M: Absence of smooth muscle actin-positive pericyte coverage of tumor vessels correlates with hematogenous metastasis and prognosis of colorectal cancer patients. Oncology 69: 159-166, 2005.

33. Gille H, Kowalski J, Li B, LeCouter J, Moffat B, Zioncheck TF, Pelletier N and Ferrara N: Analysis of biological effects and signaling properties of Flt-1 (VEGFR-1) and KDR (VEGFR-2). A reassessment using novel receptor-specific vascular endothelial growth factor mutants. J Biol Chem 276: 3222-3230, 2001.

34. Facciabene A, Peng X, Hagemann IS, Balint K, Barchetti A, Wang LP, Gimotty PA, Gilks CB, Lal P, Zhang L and Coukos G: Tumour hypoxia promotes tolerance and angiogenesis via CCL28 and $\mathrm{T}_{\text {req }}$ cells. Nature 475: 226-230, 2011.

35. Kani K, Malihi PD, Jiang Y, Wang H, Wang Y, Ruderman DL, Agus DB, Mallick P and Gross ME: Anterior gradient 2 (AGR2): Blood-based biomarker elevated in metastatic prostate cancer associated with the neuroendocrine phenotype. Prostate 73: 306-315, 2013.

36. Chen R, Pan S, Duan X, Nelson BH, Sahota RA, de Rham S, Kozarek RA, McIntosh M and Brentnall TA: Elevated level of anterior gradient-2 in pancreatic juice from patients with pre-malignant pancreatic neoplasia. Mol Cancer 9: 149, 2010.

37. Chung K, Nishiyama N, Yamano S, Komatsu H, Hanada S, Wei M, Wanibuchi H, Suehiro S and Kakehashi A: Serum AGR2 as an early diagnostic and postoperative prognostic biomarker of human lung adenocarcinoma. Cancer Biomark 10: 101-107, 2011.

38. Tohti M, Li J, Tang C, Wen G, Abdujilil A, Yizim P and Ma C: Serum AGR2 as a useful biomarker for pituitary adenomas. Clin Neurol Neurosurg 154: 19-22, 2017.

39. Di Maro G, Salerno P, Unger K, Orlandella FM, Monaco M, Chiappetta G, Thomas G, Oczko-Wojciechowska M, Masullo M, Jarzab B, et al: Anterior gradient protein 2 promotes survival, migration and invasion of papillary thyroid carcinoma cells. Mol Cancer 13: 160, 2014.

40. Mostert B, Sieuwerts AM, Bolt-de Vries J, Kraan J, Lalmahomed Z, van Galen A, van der Spoel P, de Weerd V, Ramírez-Moreno R, Smid M, et al: mRNA expression profiles in circulating tumor cells of metastatic colorectal cancer patients. Mol Oncol 9: 920-932, 2015.

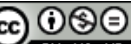

This work is licensed under a Creative Commons Attribution-NonCommercial-NoDerivatives 4.0 International (CC BY-NC-ND 4.0) License. 